\title{
Estimativa do tamanho de duas espécies de ciclídeos (Osteichthyes, Perciformes) predados por Lontra longicaudis (Olfers) (Carnivora, Mustelidae), através de análise das escamas
}

\author{
Carlos Benhur Kasper, Juliana Salvi \& Hamilton César Zanardi Grillo
}

Museu de Ciências Naturais, Centro Universitário UNIVATES. Rua Avelino Tallini 171, 95900-000 Lajeado, Rio Grande do Sul, Brasil. E-mail: felinosdosul@yahoo.com.br

\begin{abstract}
Size estimation of two species of Ciclids (Ostheichthyes, Perciformes) predated by Lontra longicaudis (Olfers) (Carnivora, Mustelidae) through scale analysis. The present study was carried out in the Taquari Valley, central region of Rio Grande do Sul State, Southern Brazil. Between December 2001 and December 2002 a study about the predation of Cichlidae by Lontra Iongicaudis (Olfers, 1818) was performed through scale analysis found in otter scats. Predation was indentified about Gimnogeophagus labiatus (Hensel, 1870) and Crenicichla punctata Hensel, 1870, and the occurence of these fish species in the diet were higher than the relative availability in the environment. A positive correlation between fish and scale sizes was found, allowing to build a regression curve to estimate the size of predated fish, based on scales found in otter scats. In this study ciclids most frequently predated varied in lenght from 100 and $150 \mathrm{~mm}$ and in weight from 22 to $37,6 \mathrm{~g}$.

KEY WORDS. Cichlidae, diet, otter, preferential predation, prey size estimation
\end{abstract}

RESUMO. O presente estudo foi realizado no Vale do Taquari, região central do Estado do Rio Grande do Sul, sul do Brasil. Entre dezembro de 2001 e dezembro de 2002 foi realizado um estudo sobre a predação de Cichlidae por Lontra longicaudis (Olfers, 1818) através de análise das escamas encontradas nas fezes de lontra. Foi identificada a predação sobre Gimnogeophagus labiatus (Hensel, 1870) e Crenicichla punctata Hensel, 1870, e a ocorrência destas espécies de peixe na dieta é mais elevada do que sua disponibilidade relativa no ambiente. Foi encontrada uma correlação positiva entre o tamanho do peixe e das escamas, permitindo a construção de uma curva de regressão para estimar o tamanho dos peixes predados baseado no tamanho das escamas encontradas nas fezes de lontra. Neste estudo, os ciclídeos mais frequentemente predados variaram no comprimento entre 100 e $150 \mathrm{~mm}$ e no peso entre 22 e $37 \mathrm{~g}$.

PALAVRAS CHAVE. Cichlidae, dieta, estimativa do tamanho de presa, lontra, predação preferencial.

Lontra longicaudis (Olfers, 1818) é um mustelídeo semiaquático encontrado do México ao Uruguai, norte da Argentina e Brasil (EISEMBERG \& REDFORD 1999), habitando rios e córregos do continente, embora possa utilizar ambientes marinhos e de água salobra (BLACHER 1987). A espécie está incluida na lista de espécies ameaçadas de extinção do Estado do Rio Grande do Sul (Fontana et al. 2003).

As lontras são animais de topo de cadeia dos ambientes onde vivem e, por isso, sujeitas a quaisquer alterações que se processem no meio (KReBs 1994). Além disto, estudos mostram que a presença de predadores pode aumentar a diversidade geral de espécies numa comunidade. Porém, os efeitos de predadores aquáticos nos ecossitemas neotropicais tem sido pouco estudados (REDFord 1997), tornando ainda mais urgente o estudo das interações destas espécies com o ambiente local.

Carnívoros em geral, têm por hábito defecar em locais conspícuos e proeminentes da sua área de vida (WEMMER et al. 1996). Este comportamento fornece um material adequado para estudos de dieta baseados na análise de conteúdo fecal, sendo amplamente utilizados no estudo da ecologia alimentar de $L$. longicaudis (Quadros \& Monteiro-Filho 2001, Colares \& Waldemarin 2000, Pardini 1998, Passamani \& Camargo 1995). Conforme estes autores, a dieta da lontra neotropical no Brasil é baseada em peixes e crustáceos, sendo complementada com outros vertebrados e invertebrados.

A família Cichlidae parece ser um importante item alimentar de L. longicaudis, tendo sido identificada em estudos de dieta desta espécie por Quadros \& Monteiro-Filho (2001) no Estado de Santa Catarina, e por Passamani \& Camargo (1995) em Minas Gerais com graus diferenciados de importância. Todavia, a única referência sobre o tamanho dos organismos predados pela lontra é apresentada por QuAdros \& MonTEIro-Filho (2001). 
O presentetrabalho visa estimar o tamanho dos ciclídeos predados por L. Iongicaudis, apresentando um método deestimativa basedo no tamanho das escamas encontradas nas fezes. Desta forma, pretende-se lançar um primeiro esforço na compreensão deste aspecto da ecologia alimentar da lontra neotropical.

\section{MATERIAL E MÉTODOS}

\section{Área de estudo}

O estudo foi realizado no rio Forqueta entre as coordenadas (UTM) 22J 3825456782080 e22J 3824006783450 , entre os municípios de São José do Herval ePutinga, área de implantação da Pequena Central Hidrelétrica Salto Forqueta. Situada na região do Vale do Taquari, porção central do Estado do Rio Grande do Sul, a área apresenta vegetação do tipo floresta estacional decidual (IBGE 1986).

A área apresenta três pontos de coleta, com aproximadamente $800 \mathrm{~m}$ de extensão, em uma seção deaproximadamente $4 \mathrm{~km}$ do rio, onde observa-se a presença de inúmeras formações rochosas e matacões de rocha nas margens. A mata ciliar da região apresenta um bom estado de conservação, exceto na área de formação do lago da barragem, onde a vegetação foi retirada para o enchimento da represa.

\section{Coleta e análise de fezes}

Foram realizadas 12 atividades a campo entre dezembro de 2001 e dezembro de 2002. Nestas saídas foram recolhidas 138 fezes de lontra para anál ise de conteúdo residual e determinação da dieta da espécie. Além disso, foram recolhidos dois restos alimentares, totalizando 140 amostras para análise da dieta.

As fezes foram acondicionadas em recipientes plásticos individuais etrazidas ao laboratório para análise. Cada amostra fecal foi lavada em água corrente sobre peneira de mal ha fina $(0,5 \mathrm{~mm})$ e conservada em recipientes plásticos com álcool a $70 \%$, para posterior triagem.

Durante a triagem manual dasfezes, foram selecionadas estruturas duras que servissem para identificar o táxon predado. Escamas e rai os duros de nadadeiras puderam fornecer subsídios suficientes para identificar as presas ao nível de família e, em alguns casos, gênero e espécie, quando comparadosa coleção de referência da área.

As escamas identificadas como sendo de representantes da família Cichlidae, foram sel ecionadas e separadas para análise morfométrica. A partir da morfometria das escamas e da comparação à coleção de referência de peixes da área de estudo, puderam ser identificadas as espécies predadas.

De cada amostrafecal anal isada, foram selecionadasaproximadamente 40 escamas. Desta amostra de escamas foram selecionadas as cinco escamas de maiores dimensões, para medição em paquímetro manual de precisão $0,05 \mathrm{~mm}$. Nesta men suração foi anal isada a maior largura decada uma das escamas sel ecionadas, obten do a largura média das maiores esca- mas da amostra, que foi submetida a uma reta de regressão, montada com base na coleção de referência de peixes da área deestudo.

\section{Coleta de peixes}

As coletas de peixes ocorreram de forma independente deste estudo, sendo uma das medidas do plano de manejo ambiental da área de construção da hidrelétrica. A amostragem de peixes foi realizada a partir da utilização de nove redes de espera, com três malhas diferentes (1.5, 2.5 e $3.5 \mathrm{~cm}$ entre nós). As redes foram mantidas por períodos de 24 horas, sendo o método aplicado em diversos pontos da área de estudo, ao longo de 11 saí das a campo, abrangen do todas as estações do ano de2001. O método de coleta foi adaptado de Benedito-Cecilio $\&$ AgostiNHO (2000).

\section{A nálise da relação entre tamanho do peixe e largura das esca} mas

A relação entre o comprimento do peixe e a largura das escamasfoi realizada com as espécies identificadas nas fezes de L. Iongicaudis. Foram utilizados 38 indivíduos (26 Gimnoge phagus labiatus (Hensel, 1870) e 12 Crenicichla punctata Hensel, 1870) da coleção do Museu deCiências Naturais da UNIVATES. O número de indivíduos de cada uma das espécies utilizado, representa a quantidade de espécimes necessários para amostrar a variação de comprimento dos peixes na coleção de referência disponível. De cada espécime selecionado foram retiradas aproximadamente 40 escamas de diferentes partes do corpo do indivíduo, sempre próximos à linha lateral, onde existe menor variação de tamanho e forma em relação às porções ventral e dorsal. Assim, obteve-se uma amostragem semelhanteàquela obti da pel a separação de 40 escamas das fezes coletadas em campo. De forma análoga ao método adotado para medição das escamas en contradas nas fezes, foram separadas as cinco escamas com maiores dimensões das quais, foi obtida uma média da largura máxima. Assim, foi criada por regressão foi criada uma equação de reta, a fim de estimar o tamanho do peixe a partir de suas escamas (ZAR 1999).

Para avaliar a eficiência das estimativas do tamanho dos peixes predados através do uso das escamas foi realizado um teste utilizando 10 indivíduos de cada espécie, presentes na coleção de referência. No teste, foi comparado pelo teste t de Student (ZAR 1999) o tamanho estimado do peixe (calculado a partir da equação de reta criada) ao tamanho real do peixe do qual foram retiradas as escamas.

\section{A nálise sobre a predação de cidídeos}

Primeiramente foram determinadas quais as espécies de ciclídeos predadas por L. longicaudis. A partir da identificação dostáxons, foram comparadas as diferenças entre a predação de diferentes classes de tamanho e entre as espécies. Neste estudo foram adotadas três classes detamanho: $<100 \mathrm{~mm}, 100 \mathrm{a}$ $150 \mathrm{~mm}$ e $>150 \mathrm{~mm}$. A freqüência de ocorrência em cada classe de tamanho foi comparada entre os indivíduos predadose 
àqueles amostrados na área deestudo, para inferências acerca da possibilidade de existência de seletividadede. A partir da estimativa decomprimento dos indivíduos predados, foi possível obter uma estimativa do peso médio das presas. Esta estimativa foi real izada a partir da obtenção de uma média de peso em uma subamostragem de dez indivíduos, da coleção de referência, dentro da classe de tamanho desejada.

\section{RESULTADOS}

\section{Coleta e análise das fezes}

Os peixes estiveram presentes em $97,1 \%$ das amostras, onde os ciclídeos foram o segundo grupo de peixes mais comum, presentes em 47,9\% das fezes triadas (67 amostras), superado apenas pela família Loricariidae, presentes em 69,3\% dasamostras.

Duas espécies de ciclídeos foram identificadas: Gimnogephagus labiatus (popularmenteconhecido como "cará") eC renicichla punctata (popularmente conhecido como "joana"), presentes em 27,9 e 20,0\% das amostras, respectivamente.

\section{Coleta de peixes}

No estudo da ictiofauna local foram coletados 2625 indivíduos, sendo 3,6\% destes representantes da família Cichlidae. Entre os ciclídeos, os mais abundantes foram G. labiatus e C. punctata, respectivamente com 2,4 e 1,2\% dos espécimes coletados

\section{A nálise da relação entre o tamanho do peixe e largura das escamas}

Para G. Iabiatus obteve-sea seguinte equação de reta, através de regressão: $Y=0,2+0,43 . X$, onde $Y$ é o tamanho da escamaeX o tamanho do peixe. No teste t aplicado afim deavaliar a precisão da reta de regressão, foi obtido $t=0,1740$ (18 G.L., $p$ $=0,864$ N.S.), indicando que o tamanho estimado do peixe não varia significativamente de seu tamanho real.

Para C. punctata obteve-sea seguinteequação dereta através de regressão: $Y=-0,16+0,02 . X$, onde $Y$ é o tamanho da escama e $X$ o tamanho do peixe. Para o teste t realizado foi obtido $t=0,8925$ (18 G.L., $p=0,918$ N.S.), indicando que $o$ tamanho estimado do peixe não varia significativamente de seu tamanho real.

\section{A nálise sobre a predação de cidídeos}

Comparando a freqüência de ocorrência nas fezese a disponibilidade observada no ambiente, nota-se que existe seletividade na dieta, uma vez que os índices de predação sobre estas espécie são maiores do que sua disponibilidade relativa no ambiente (Fig. 1).

Os espécimes deG. labiatus predadosapresentaram comprimento variando entre $87,4 \mathrm{~mm}$ e $207,0 \mathrm{~mm}$, enquanto que os espécimes de $C$. punctata variaram entre $77,0 \mathrm{~mm}$ a $318,5 \mathrm{~mm}$. Observou-se que, em ambas as espécies, os peixes mais frequentemente predados possuiram entre 100 e $150 \mathrm{~mm}$ (Figs 2 e 3). $O$ peso médio dos peixes observado nesta classe de

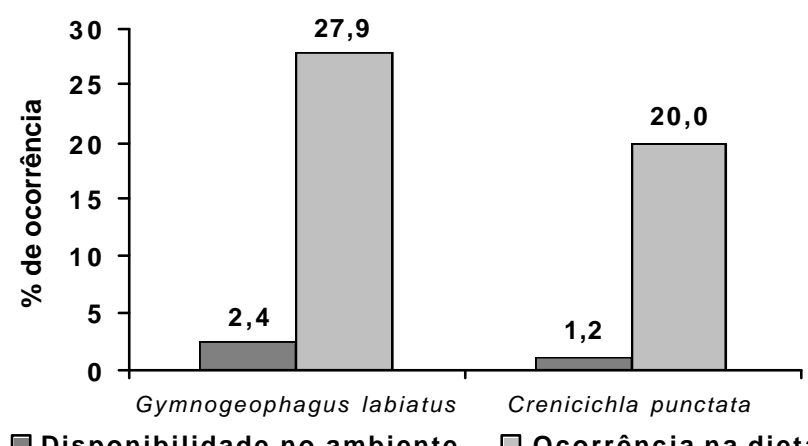

$\square$ Disponibilidade no ambiente $\square$ Ocorrência na dieta Figura 1. Relação entre disponibilidade relativa no ambientee a ocorrência de ciclídeos na dieta de Lontra longicaudis no Rio Forqueta, Vale do Taquari, Rio Grande do Sul.

tamanho foi de $37,6 \mathrm{~g}( \pm 6,1 \mathrm{~g})$ paraG. labiatus, e $22,0 \mathrm{~g}( \pm 4,7 \mathrm{~g})$ para C. punctata.

Relacionando a disponibilidade relativa no ambienteea freqüência com que aparecem nas fezes, nota-se queG. labiatus medindo entre 100 e 150 mm, são consumidos em proporções maiores do que o esperado (Fig. 2).
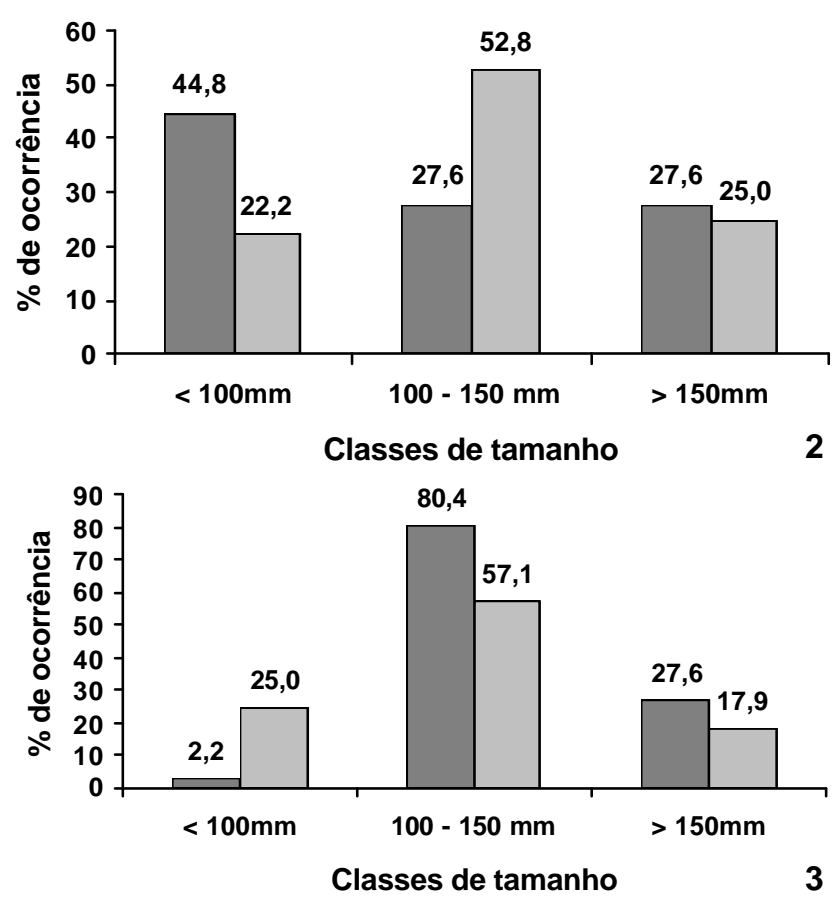

$\square$ Disponibilidade no ambiente $\square$ Ocorrência na dieta

Figuras 2-3. Relação entre a disponibilidade relativa no ambiente e a predação de: (2) Gymnogeophagus labiatus e (3) Crenicichla punctata, por classe detamanho, no Rio Forqueta, Valedo Taquari, Rio Grande do Sul. 
As relações entre disponibilidade relativa no ambientee a freqüência de ocorrência nas fezes, de cada classe de tamanho em C. punctata, podem ser observadas na figura 3.

\section{DISCUSSÃ O}

O método adotado neste estudo visa estimar o tamanho de peixes predados por lontras, com base nos restos al imentares não digeridos encontrados nas fezes da espécie. Para tanto, foi desenvolvido um método que utiliza o tamanho das escamas de duas espécies de ciclídeos amplamente consumidas, como base de estudo.

Este método leva em consideração as maiores escamas de cada amostra, nas estimativas de tamanho da presa. A utilização das escamas de mai ores dimensões foi adotada pois existevariação no tamanho das escamas (geral mente maiores próximos a linha lateral e menores na região ventral e dorsal do peixe). Assim, a utilização de escamas al eatoriamente escolhidas poderia resultar em uma subestimativa do tamanho dos peixes predados. Desafortunadamente, o método exclui peixes menores que tenham sido predados e estejam presentes na mesma amostra fecal com um peixe maior, uma vez que não foi encontrada uma maneira segura de diferenciar as escamas pequenas de um indivíduo grande, das maiores escamas de um indivíduo pequeno, se estas estiverem na mesma amostra.

A utilização de redes de espera na amostragem de peixes da área de estudo pode ser considerada seletiva, pois captura apenas os indivíduos que nela colidem durante seus deslocamentos. Todavia, o método é consagrado em estudos de ictiologia, sendo amplamenteutilizado para a obtenção de dados básicos da composição da comunidade de peixes em diversos ambientes, notadamente deambientes lóticos. Não obstante, a facilidade e baixo custo de aplicação do método, justificam sua utilização para obtenção dos dados bási cos a que este estudo se destina.

A predação sobreciclídeosé conhecida paraL. Iongicaudis, tendo sido citada por autores como PasSam ANI \& CAMARGO (1995) e Quadros \& M onteiro -Filho (2001). Passamani \& Camargo (1995) citam a ocorrência da família Cichlidae em $88,9 \%$ das amostras analisadas em seu estudo, destacando a presença de Geophagus brasiliensis e Tilapia rendalli (espécie exótica introduzida no Brasil). Quadros \& M onteIro-FILHo (2001) citam a ocorrência de Geophagus brasiliensis em $27,7 \%$ das amostras, sendo este o único ciclídeo identificado.

No presenteestudo, foi identificada a predação sobreoutras duas espécies de ciclídeos: Gimnogephagus labiatus e Crenicichla punctata, sendo que a freqüência de predação destes ciclídeos mostrou-se intermediária aos dois trabal hos citados anteriormente, ocorrendo em 47,9\% das amostras analisadas.

As estimativas do tamanho das presas utilizadas pela lontra foram feitas a partir de métodos estatísticos básicoseforne ceram uma idéia aproximada do tamanho de presas utilizado por L. Iongicaudis. A partir destas análises, foi possível constatar quea predação ocorreu principalmente sobre indivíduos de ta- manho médio (entre $100 \mathrm{~mm}$ e $150 \mathrm{~mm}$ ). O peso aproximado dos peixes predados é de $37,6 \mathrm{~g}( \pm 6,1 \mathrm{~g})$ paraG. labiatus e 22,0 $\mathrm{g}( \pm 4,7 \mathrm{~g})$ paraC. punctata nos indivíduos da classedetamanho citado. Os valores obtidos no presente estudo diferem muito dos valores citados por Quadros \& M ONTEIRO-FILHo (2001), que citam um peso médio de $350 \mathrm{~g}$ para os peixes predados.

Destaca-se que hove um indicativo de predação preferencial pelos peixes da família Cichlidae, uma vez que sua freqüência de ocorrência nas fezes foi muito mais el evada do que a disponibilidade rel ativa observada no ambiente. Verificou-se ainda, que os peixes com tamanho entre $100 \mathrm{~mm}$ e $150 \mathrm{~mm}$ foram os mai s predados, eque houve uma predação preferencial sobre esta classe de tamanho no caso de G. labiatus.

Em relação aC. punctata a classedetamanho maispredada é também a mais abundante. Verifica-se uma predação maior do que a disponibilidade observada no ambiente dos peixes desta espécie menores do que $100 \mathrm{~mm}$, embora este valor possa estar distorcido, uma vez que as redes de espera possuiam malhas muito grandes para captura desta espécie nesta classe detamanho.

Em suma, verificou-se que a família Cichlidae apresenta grande importância na dieta de Lontra longicaudis na área de estudo. Notou-se que houve seletividade na predação deste grupo, não somente em relação as diferentes espécies de peixes, mas também em função do tamanho das presas. Contudo, énecessário o aprofundamento dos estudos em questão.

Os autores sugerem que o método adotado neste estudo deva ser discutido, aprimorado e adaptado também a outros taxa, vizando a obtenção de um método mais preciso e padronizado de estudo.

\section{A GRADECIMENTOS}

Agradecemos ao Biológo M arco Antônio Majolo ecolaboradores (levantamento ictiológico), Dr. Eduardo Périco (revisão e estatística), Dra. Susi Missel Pacheco (revisão final ), M Sc. Helen Waldemarin, a Bióloga MariaJúlia Feldense amigos pelo apoio, amizadee companherismo durante toda a execução do projeto. Especial agradecimento ao Centro Universitário UNIVATES E CERTEL pelo apoio e financiamento ao projeto.

\section{REFERÊNCIAS BIBLIOGRÁ FICAS}

Benedito-Cecilio, E. \& A.A. Agostinho. 2000. Distribution, abundance and use of different environments by dominant ichthyofauna in the influence area of Itaipu Reservoir. Acta Scien tiarum, M aringá, 22 (2): 429-437.

BLACHER, C. 1987. Ocorrência e preservação de Lutra longicaudis (Mammalia: Mustelidae) no litoral de Santa Catarina. Boletim da Fun dação Brasil ei ra para Conservação da Natureza, Rio de Janeiro, 22: 105-117.

Colares, E.P. \& H.F. W aldemarin . 2000. Feeding of theneotropical river otter (Lontra Iongicaudis) in the costal region of the Rio Grande do Sul State, Southern Brazil. IUCN Otter 
Specialist Group Bull, Wageningen, 17: 6-13.

Eisemberg, J.F. \& K.H. Redford. 1999. Mammals of the Neotropics: The Central Neotropics. Chicago, University of Chigago Press, 609p.

Fontana, C.S.; G.A. Bencke \& R.E. Reis 2003. Livro Vermel ho das Espécies da Fauna Ameaçada de Extinção no Rio Grande do Sul. Porto Alegre, EDIPUCRS, 632p.

IBGE. 1986. Levantamento de Recursos Naturai s: Ecologia, Geomorfologia, Pedologia, Vegetação, Uso potencial da Terra. IBGE, Rio de Janeiro, Folha S.H. 22, p. 541-632.

KREBS, C.J. 1994. Ecology. California, Harper Colins, $4^{\text {th }}$ ed., 801p.

PARDINI, R. 1998. Feeding ecology of the neotropical river otter Lontra Iongicaudis in Atlantic Forest strem, south-estern Brazil. Journal of Zoology, London, 245: 385-391.

Passamani M. \& S.L. Camargo. 1995. Diet of the river otter Lutra Iongicaudis in Furnas Reservoir, south-eastern Brazil. Otter
Specialist Group Bulletin, Wageningen, 12: 32-34.

Quadros, J. \& E.L.A. Monteiro-Filho. 2001. Diet of the Neotropical Otter, Lontra Iongicaudis, in an Atlantic Forest Area, Santa Catarina State, Southern Brazil. Studi es of the Neotropical Faunaand Environment, Nisse, 36: 15-21.

Redford, K.H. 1997. A Floresta Vazia, p. 1-22. In: C. VallaresPÁdua \& R.E. Bodmer (Eds). M anejo e Con servação de Vida Silvestre no Brasil. Belém, MCT, CNPq, Sociedade Civil Mamirauá, 285p.

Wemmer, C.; T.H. Kuns; G. Lundie-Jenkins \& W.J. Mcshea. 1996. Mammalian Sign, p. 157-176. In: D.E. W ILSON; F.R. CoLE; J.D. Nichols; R. Rudan \& M.S. Foster. (Eds). Mensuring and Monitoring Biological Diversity, Standard Methodsfor Mammals. Washington, Smithsonian Institution Press, 409p.

$\mathrm{ZAR}_{\mathrm{AR}}$ J.H. 1999. Biostatistical Analysis. NewJersey, PrenticeHall, $4^{\text {th }}$ ed., 663p.

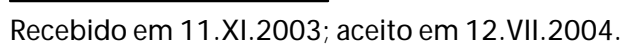

